\title{
Inverted stress cardiomyopathy during monitored anesthesia care for endoscopic sinus surgery
}

\author{
Min Seok Koo ${ }^{1}$, Jung Won Lee ${ }^{1}$, Do-Hun Kim', Jung Ju Sir ${ }^{2}$, and Jung Min Kim ${ }^{1}$ \\ Departments of ${ }^{1}$ Anesthesiology and Pain Medicine, ${ }^{2}$ Internal Medicine, National Medical Center, Seoul, Korea
}

A 51-year-old man (height, $169 \mathrm{~cm}$; weight, $64 \mathrm{~kg}$ ) visited the hospital for endoscopic sinus surgery to treat chronic sinusitis. His past medical history and pre-operative evaluation were unremarkable. The surgeon and patient requested monitored anesthesia care (MAC) because the lesion was minor and the patient was reluctant to undergo general anesthesia. Therefore, the surgery was scheduled to be under MAC with a patient controlled analgesia (PCA) device. No premedication was administered. On arrival at the operating room, the patient's initial vital signs were within normal limits, and his electrocardiogram (ECG) showed normal sinus rhythm. The PCA device used was an ambulatory infusion pump (Accumate ${ }^{\circledR} 1000$; Wooyoung Medical, Seoul, Korea) and contained a combination of $200 \mathrm{mg}$ $1 \%$ propofol, $100 \mu \mathrm{g}$ fentanyl, and $30 \mathrm{mg} 2 \%$ lidocaine; the flow rate was set at $10 \mathrm{ml} / \mathrm{h}$ with a bolus of $0.5 \mathrm{ml}$, and a lock-out time of $1 \mathrm{~min}$. The surgery started with an injection of $3 \mathrm{ml}$ of $1: 100,000$ epinephrine into the nasal cavity. The patient's vital signs were stable, but he complained of slight discomfort and anxiety during the surgery. Twenty-five minutes later, the patient complained of chest tightness; his blood pressure (BP) was 90/45 mmHg, peripheral oxygen saturation $\left(\mathrm{SpO}_{2}\right)$ was $89 \%$, and the ECG showed a sinus tachycardia of 170 beats/min. Twenty milligrams of esmolol was administered, and his BP immediately dropped to $85 / 40 \mathrm{mmHg}$, his heart rate (HR) was $170-$ 180 beats/min, he had an $\mathrm{SpO}_{2}$ of $85 \%$, and the ECG showed a monomorphic ventricular tachycardia. The surgery and PCA were stopped, $100 \%$ oxygen was administered through an oxygen mask, and $120 \mathrm{mg}$ lidocaine was injected intravenously. The ECG showed a reversion to normal sinus rhythm; however, an
ST-segment depression was observed, his BP was 90/50 mmHg, $\mathrm{HR}$ was 90 beats/min, and $\mathrm{SpO}_{2}$ was $85 \%$. An intravenous injection of $10 \mathrm{mg}$ ephedrine was administered, and an intravenous continuous infusion of nitroglycerine $(0.3 \mu \mathrm{g} / \mathrm{kg} / \mathrm{min})$ was started. Approximately $3 \mathrm{~min}$ later, his BP reached 140/90 $\mathrm{mmHg}$, $\mathrm{HR}$ increased to 100 beats/min, and $\mathrm{SpO}_{2}$ improved to $98 \%$. On echocardiography, the left ventricular ejection fraction was $45 \%$, and akinesia of the left ventricle basal and mid-ventricular segments were observed (Fig. 1). The coronary angiography results showed no coronary artery obstruction. Owing to the normal ECG prior to surgery, the presence of physical and emotional stress, and neither myocarditis nor pheochromocytoma in the medical history, a diagnosis of inverted stress cardiomyopathy was made. Troponin I values were $6.34 \mathrm{ng} / \mathrm{ml}$ on the day of surgery, $1.93 \mathrm{ng} / \mathrm{ml}$ one day postoperatively, and $0.20 \mathrm{ng} / \mathrm{ml}$ two days postoperatively. On the second postoperative day, the left ventricular ejection fraction on echocardiography had increased to $65 \%$, without evidence of hypokinesia.

Stress cardiomyopathy is characterized by transient left ventricular regional wall motion abnormalities without coronary artery obstruction and occurs more frequently in postmenopausal women. Recently, in contrast to typical stress cardiomyopathy, there have been reports of variations in motion abnormalities in the basal or mid-ventricular segments while retaining apical mobility. In stress cardiomyopathy, excess catecholamines play an important role in the pathophysiology. The large distribution of beta-adrenergic receptors in the apex renders it vulnerable to an overload of catecholamines, which develops into a typical stress cardiomyopathy. The presence of morphological variants

\footnotetext{
Corresponding author: Min Seok Koo, M.D., Ph.D., Department of Anesthesiology and Pain Medicaine, National Medical Centar, 18-79, Euljiro 6-ga, Jung-gu, Seoul 100-799, Korea. Tel: 82-2-2260-7373, Fax: 82-2-2262-4766, E-mail: facharzt@gmail.com

(c) This is an open-access article distributed under the terms of the Creative Commons Attribution Non-Commercial License (http:// creativecommons.org/licenses/by-nc/3.0/), which permits unrestricted non-commercial use, distribution, and reproduction in any medium, provided the original work is properly cited.
} 

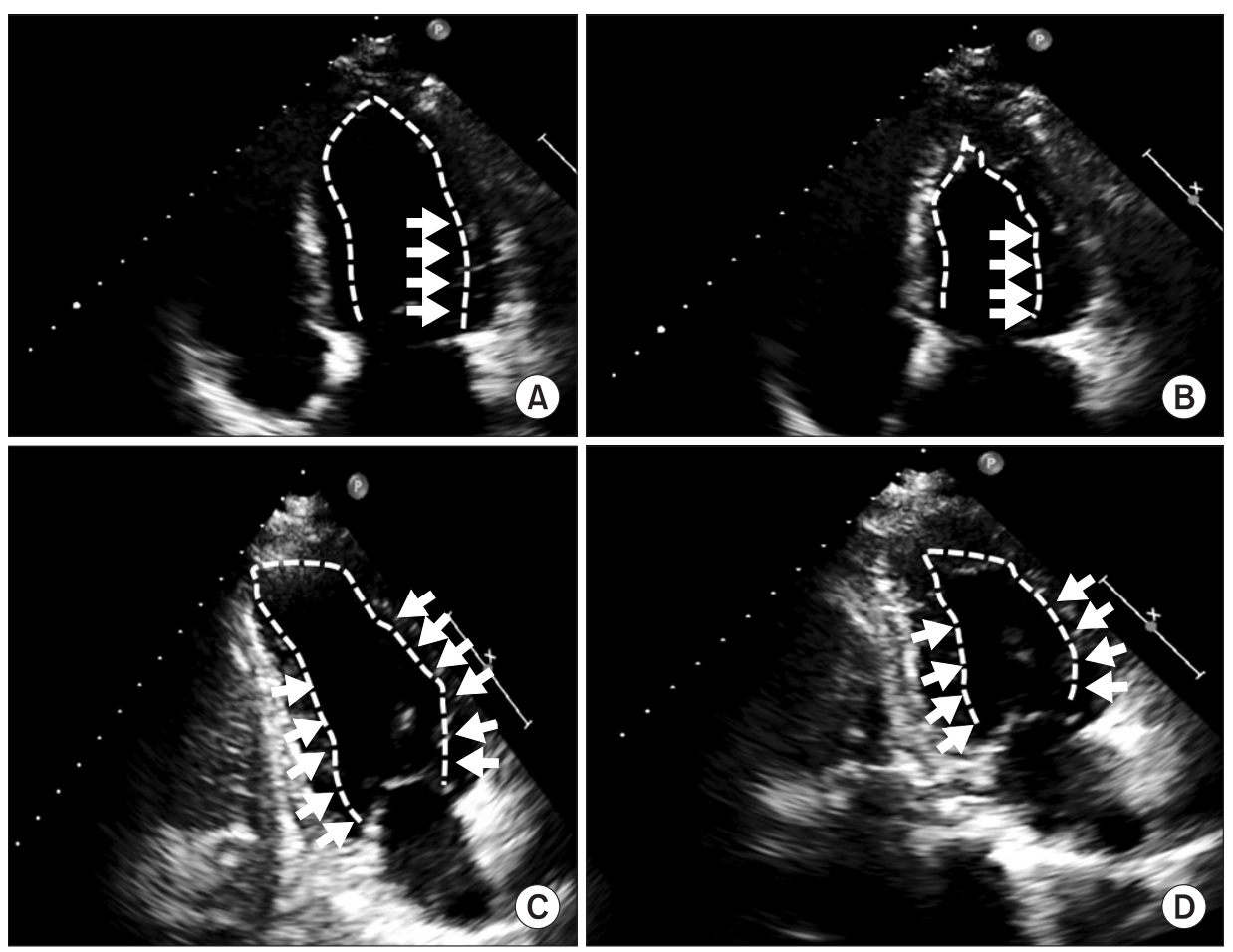

Fig. 1. The transthoracic echocardiogram showed akinesia of anterolateral, inferior and anterior wall of left ventricle (white arrow). (A) diastole and (B) systole at apical four chamber view; (C) diastole and (D) systole at apical two chamber view.

in stress cardiomyopathy can be expected due to the anatomical variation in the sympathoadrenergic system and the distribution of beta-adrenergic receptors [1]. Chest pain is the most common symptom of stress cardiomyopathy, but dyspnea and pulmonary edema can also be present [2]. There are various predisposing factors for stress cardiomyopathy, including psychological stress, administration of catecholamines, sepsis, intracranial hemorrhage, and surgery [3]. The diagnostic criteria suggested by the Mayo clinic are (1) temporary wall motion abnormalities involving the apical or mid-ventricular segment of the left ventricle exceeding the area of a single coronary artery, (2) no coronary artery obstruction, (3) new ECG abnormalities such as ST-segment elevation or T-wave inversion, or modest increase of cardiac troponin levels, and (4) exclusion of myocarditis or pheochromocytoma [4]. There are no established guidelines for treating stress cardiomyopathy; treatment is based on clinical conditions. The use of angiotensin-converting enzyme inhibitors is suggested for the left ventricular systolic dysfunction, and beta and alpha blockers can be considered to prevent relapse. If hypotension is present, the patient should be screened for left ventricular outflow tract (LVOT) obstruction [3].

In the perioperative period, stress cardiomyopathy should be considered in patients presenting with chest pain, dyspnea, pulmonary edema, ECG changes mimicking acute coronary syndrome, or cardiogenic shock after a psychologically or physically stressful event. The diagnosis depends upon regional wall motion abnormalities that exceed the area of a single coronary artery and the lack of evidence for acute coronary syndrome [4]. Intraoperative management is mainly supportive and involves hydration and stress relief. In cases of hypotension without LVOT, inotropic agents and an intra-aortic balloon pump are suggested. If LVOT obstruction is present, fluid resuscitation, beta blockers, and phenylephrine administration are recommended [3]. Catecholamines should be used cautiously [5]. It is difficult to sufficiently prevent anxiety and pain using a PCA device because bolus doses of sedatives and analgesics are administered after the onset of anxiety and pain. Consequently, the likelihood of stress cardiomyopathy might be increased in these cases. Therefore, anesthesiologists should be fully aware of the stress resulting from surgical manipulation and the patient's status. It is important to manage the patient's pain and anxiety appropriately before the onset of psychological and physical stress, especially in those susceptible to stress cardiomyopathy [5]. Patients under MAC can express their symptoms. MAC is superior to general anesthesia for early detection of stress cardiomyopathy. However, MAC has disadvantages such as psychological and physical stress due to light anesthesia and delayed airway management. Neither MAC nor general anesthesia is superior with regard to the overall effect on stress cardiomyopathy.

The differential diagnosis is important in stress cardiomyopathy because the presenting features are similar to those of acute coronary syndrome. Therefore, anesthesiologists should be aware of the possibility of stress cardiomyopathy in order to provide proper management to the patient. 


\section{References}

1. Nef HM, Möllmann H, Akashi YJ, Hamm CW. Mechanisms of stress (Takotsubo) cardiomyopathy. Nat Rev Cardiol 2010; 7: 187-93.

2. Hurst RT, Prasad A, Askew JW 3rd, Sengupta PP, Tajik AJ. Takotsubo cardiomyopathy: a unique cardiomyopathy with variable ventricular morphology. JACC Cardiovascular Imaging 2010; 3: 641-9.

3. Bybee KA, Prasad A. Stress-related cardiomyopathy syndromes. Circulation 2008; 118: 397-409.

4. Prasad A. Apical ballooning syndrome: an important differential diagnosis of acute myocardial infarction. Circulation 2007; 115: e56-9.

5. Liu S, Dhamee MS. Perioperative transient left ventricular apical ballooning syndrome: Takotsubo cardiomyopathy: a review. J Clin Anesth 2010; 22: 64-70. 